\title{
What and Where Information in the Caudate Tail Guides Saccades to Visual Objects
}

\author{
Shinya Yamamoto, ${ }^{1,2}$ Ilya E. Monosov, ${ }^{1}$ Masaharu Yasuda, ${ }^{1}$ and Okihide Hikosaka ${ }^{1}$ \\ ${ }^{1}$ Laboratory of Sensorimotor Research, National Eye Institute, National Institutes of Health, Bethesda, Maryland 20892, and ${ }^{2}$ System Neuroscience, Human \\ Technology Research Institute, National Institute of Advanced Industrial Science and Technology (AIST), Tsukuba 305-8568, Japan
}

We understand the world by making saccadic eye movements to various objects. However, it is unclear how a saccade can be aimed at a particular object, because two kinds of visual information, what the object is and where it is, are processed separately in the dorsal and ventral visual cortical pathways. Here, we provide evidence suggesting that a basal ganglia circuit through the tail of the monkey caudate nucleus (CDt) guides such object-directed saccades. First, many CDt neurons responded to visual objects depending on where and what the objects were. Second, electrical stimulation in the $\mathrm{CDt}$ induced saccades whose directions matched the preferred directions of neurons at the stimulation site. Third, many $\mathrm{CDt}$ neurons increased their activity before saccades directed to the preferred objects and directions of the neurons in a free-viewing condition. Our results suggest that CDt neurons receive both "what" and "where" information and guide saccades to visual objects.

\section{Introduction}

In the visual association cortices, the processing of spatial ("where") information and object ("what") information is roughly segregated into the dorsal and ventral cortical visual pathways (Mishkin et al., 1983; Goodale and Milner, 1992). This hypothetical scheme poses a problem, however, when we plan to make a motor action aiming at a particular object, say, by making a saccadic eye movement. If a group of neurons in the ventral visual pathway detect the object but encode little information on its position, how can they contribute to the saccade aiming at the position of the object? One possibility is that the segregation of information processing is not strict: object-selective neurons in the inferotemporal cortex may carry position information (Op De Beeck and Vogels, 2000; DiCarlo and Maunsell, 2003; Sereno and Lehky, 2011), position-encoding neurons in the parietal or frontal cortex may carry object information (Sereno and Maunsell, 1998; Murata et al., 2000; Janssen et al., 2008; Peng et al., 2008), or the position and object information may converge in the prefrontal cortical areas (Rao et al., 1997; Rainer et al., 1998). It is unclear, however, whether such convergent information is accurate enough to enable motor actions aiming at particular objects.

One alternative mechanism underlying such "object-directed actions" may be the neural circuits through the basal ganglia.

Received Feb. 20, 2012; revised June 2, 2012; accepted June 3, 2012.

Author contributions:S.Y., I.E.M., M.Y., and 0.H. designed research;S.Y., I.E.M., and 0.H. performed research;S.Y. and 0. H. analyzed data; S.Y., I.E.M., M.Y., and O.H. wrote the paper.

This work was supported by the intramural research program at the National Eye Institute. We thank E. S. Bromberg-Martin, S. Hong, H. Kim, and Y. Tachibana for valuable discussions, and A. Hays, J. W. McClurkin, B. Nagy, A. M. Nichols, D. Parker, T. W. Ruffner, M. K. Smith, G. Tansey, N. Phipps, C. Zhu, F. Ye, and D. Leopold for technical assistance.

This article is freely available online through the J Neurosci Open Choice option.

Correspondence should be addressed to Shinya Yamamoto, Human Technology Research Institute, National Institute of Advanced Industrial Science and Technology (AIST), 1-1-1, Umezono, Tsukuba 305-8568, Japan. E-mail: yamamoto-s@aist.go.jp.

DOI:10.1523/JNEUROSCI.0828-12.2012

Copyright $\odot 2012$ the authors $\quad 0270-6474 / 12 / 3211005-12 \$ 15.00 / 0$
Studies using behaving monkeys have shown that the basal ganglia nuclei contain neurons that respond to visual stimuli in a position-selective manner (Hikosaka et al., 2000) and may control motor actions by sending the position information to the superior colliculus (Hikosaka and Wurtz, 1983b; Karabelas and Moschovakis, 1985; Liu and Basso, 2008). The position information may be derived from the dorsal cortical visual pathway, which heavily projects to the anterior part of the striatum, particularly the head and body of the caudate nucleus (Selemon and Goldman-Rakic, 1985; Stanton et al., 1988; Shook et al., 1991; Parthasarathy et al., 1992).

On the other hand, visual object information is transmitted predominantly to the tail of the caudate nucleus (hereafter referred to as $\mathrm{CDt}$ ). Highly developed in the primate (including humans and macaque monkeys), the CDt is a long caudal extension of the caudate nucleus, lying along the temporal lobe (see Fig. $1 A, B$ ). Consistent with this anatomical position, the CDt receives inputs mainly from the temporal cortex, particularly cortical areas in the ventral visual pathway (Yeterian and Van Hoesen, 1978; Van Hoesen et al., 1981; Saint-Cyr et al., 1990; Webster et al., 1995; Middleton and Strick, 1996). Neurons in the CDt respond to visual stimuli in an objectselective manner (Caan et al., 1984; Brown et al., 1995). Furthermore, the CDt projects to the dorsolateral part of the substantia nigra pars reticulata (SNr) (Saint-Cyr et al., 1990), which contains many neurons projecting to the superior colliculus (SC) (Beckstead et al., 1981). However, it is unclear whether CDt neurons encode position information.

We found in behaving monkeys that CDt neurons encoded not only what but also where information of visual objects, weak electrical stimulation in the CDt induced saccades, and CDt neurons became active before saccades to particular positions and particular objects.

\section{Materials and Methods}

We used two male rhesus monkeys (Macaca mulatta), Z and T. After each monkey was sedated by general anesthesia, we implanted a head holder, 
a chamber for unit recording, and eye coils. All animal care and experimental procedures were approved by the National Eye Institute Animal Care and Use Committee and complied with the Public Health Service Policy on the humane care and use of laboratory animals.

\section{Behavioral tasks}

Behavioral tasks were controlled by a custom real-time experimentation data acquisition system (REX, Laboratory of Sensorimotor Research, National Eye Institute-National Institutes of Health, Bethesda, MD) (Hays et al., 1982). Two monkeys ( $\mathrm{Z}$ and $\mathrm{T}$ ) participated in the experiments. The monkeys sat in a primate chair and faced a front screen on which visual stimuli were presented.

Fractals used as visual objects. We created visual stimuli using fractal geometry (Miyashita et al., 1991). One fractal was composed of four point-symmetrical polygons, which were overlaid around a common center such that smaller polygons were positioned more front. The parameters that determined each polygon (size, edges, color, etc.) were chosen randomly. Its size was $\sim 8 \times 8^{\circ}$. We used a fixed set of eight fractals (hereafter called "standard set of fractals") to examine all CDt neurons recorded. In addition to this standard set of fractals, we used many other sets of fractals. This allowed us to examine the responses of CDt neurons to fractals with different familiarity, particularly novel fractals (which the monkey had never seen before).

Object-directed saccade task. The purpose of this task was to examine the object and position selectivity of CDt neurons. The monkey made a saccade to a fractal object to obtain a fixed amount of liquid reward (see Fig. 2 A). The target object was chosen from a standard set of eight fractals (see Fig. 2C, top) and was presented at one of five positions (see Fig. 2B; right, left, up, and down with the eccentricity of $15^{\circ}$, and the center). The task started with the presentation of a central spot of light [fixation point (FP)] on which the monkey had to fixate. After $700 \mathrm{~ms}$, a fractal target (TG) was presented, but the monkey was required to keep fixating on the fixation point. After an FP-TG overlap period ( $600 \mathrm{~ms}$ for monkey Z; $450 \mathrm{~ms}$ for monkey $\mathrm{T}$ ), the FP disappeared as a GO signal to make a saccade to the TG. When TG was presented at the center, the monkey was not required to make any saccade. The criteria of fixation were $\pm 4^{\circ}$ for FP and $\pm 7^{\circ}$ for TG. Therefore, when the TG was presented on the FP, the window of the criterion became large. The monkey received a liquid reward after holding its gaze on the TG for $600 \mathrm{~ms}$. The trial was aborted if the monkey broke fixation. After the aborted trial, the same target was presented on the next trial. The target was chosen pseudorandomly such that, within a subblock of 40 trials, the 8 fractals and the 5 positions were randomized. For each neuron, at least 2 blocks ( 80 trials) were tested (range, 2-11 blocks; median, 6 blocks).

Passive-viewing task. The purpose of this task was to examine the object selectivity of the visual responses of CDt neurons. Fractal objects were presented while the monkey was fixating on the central FP. On each trial, two to six fractals were chosen pseudorandomly from the standard set of eight fractals and were presented in sequence at the preferred position of the recorded neuron. Each fractal was presented for $400 \mathrm{~ms}$, followed by a 500-700 ms blank period. The presentation of the fractals started 600$800 \mathrm{~ms}$ after the monkey started fixating on the FP. A liquid reward was given 500-700 ms after the presentation of the last fractal, but it was not associated with particular objects. The preferred position of the neuron
Table 1. 0bject and position selectivity of visual responses in the caudate tail

\begin{tabular}{llll}
\hline & \multicolumn{2}{l}{ Position selectivity } & \\
\cline { 2 - 3 } & Yes & No & \\
\hline Object selectivity & & & \\
Yes & $76(77 \%)(Z, 61 ; T, 15)$ & $4(4 \%)(Z, 2 ; T, 2)$ & $80(81 \%)$ \\
No & $17(17 \%)(Z, 13 ; T, 4)$ & $2(2 \%)(Z, 2 ; T, 0)$ & $19(19 \%)$ \\
All & $93(94 \%)$ & $6(6 \%)$ & $99(100 \%)$ \\
\hline
\end{tabular}
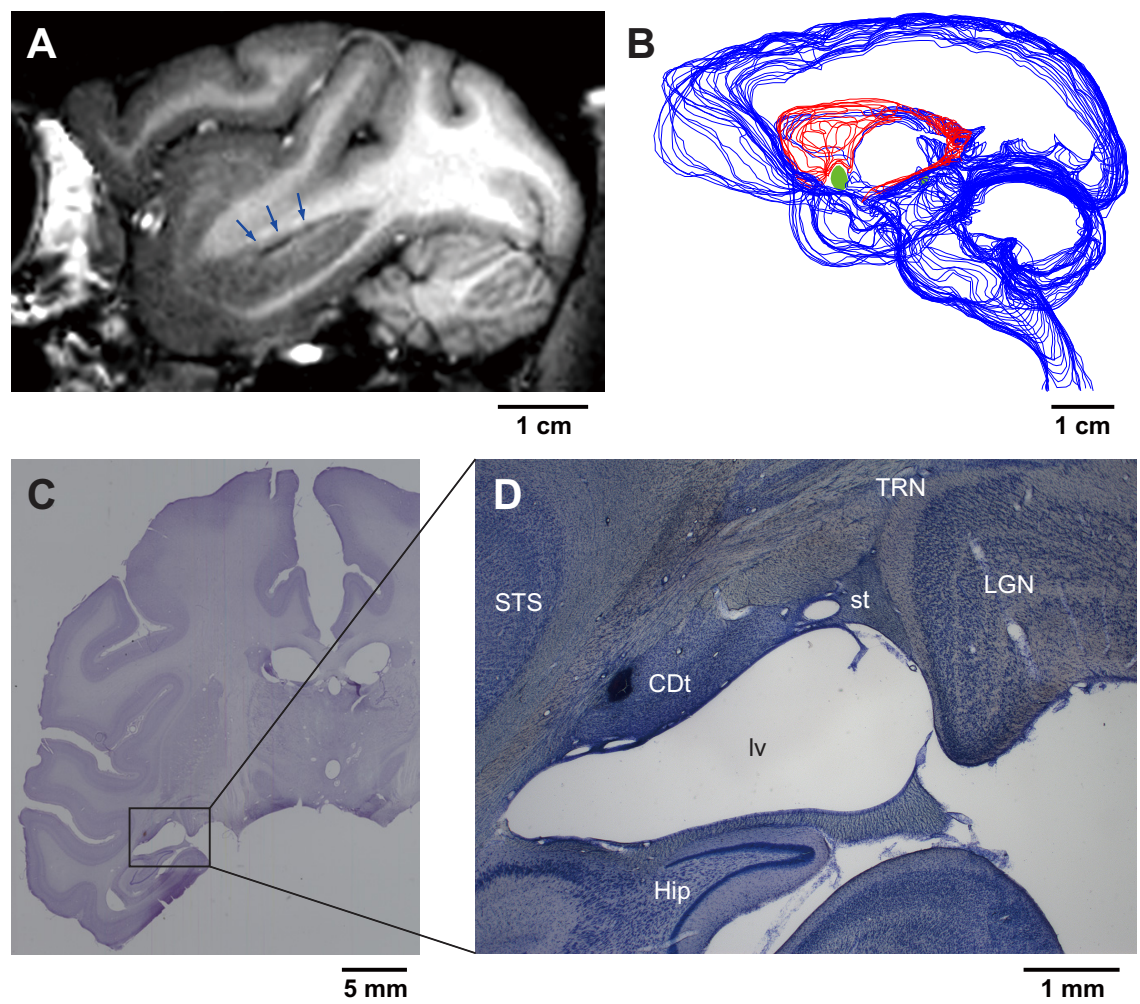

Figure 1. Anatomy of the CDt. $A$, An MR image including the caudate tail. The image plane is tilted laterally by $25^{\circ}$ in which most of the electrode tracks to the CDt are included. The CDt is indicated by arrows. Below the CDt is the lateral ventricle (thin black portion) and the 政

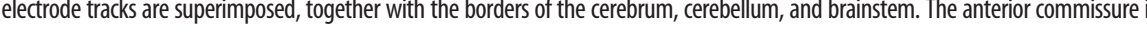
surrounded by the posterior part of the putamen (Put), lateral geniculate nucleus (LGN), thalamic reticular nucleus (TRN), stria terminalis (st), lateral ventricle (Iv), hippocampus (Hip), and fundus of the superior temporal sulcus (STS).

was determined by presenting the fractals at different positions before recording or using the object-directed saccade task. In addition to the standard set of eight fractals, we used novel object set to compare the visual response to novel and familiar objects.

Free-viewing condition. The purpose of this task was to examine the activity of CDt neurons related to gaze orienting behavior in the presence of multiple visual objects. On every trial, four fractals were presented simultaneously at four radially symmetrical positions (i.e., positions shown in Fig. $12 \mathrm{~A}$, or tilted by $45^{\circ}$ ) with the eccentricity of $15^{\circ}$. On each odd-numbered trial, four fractals were chosen randomly from a set of eight fractal objects. On the following even-numbered trial, the remaining four fractals were presented. Each fractal presentation lasted for 3000 $\mathrm{ms}$, which was followed by a $500-700 \mathrm{~ms}$ blank period before the next fractal presentation. During or between fractal presentations, no reward was delivered and the monkey was not required to do anything. Nonetheless, the monkey looked at the fractals by making saccades frequently from one fractal to another. The frequency of the saccades during the fractal presentation was $2.0 \pm 0.7$ times per second (mean $\pm \mathrm{SD}$ ), which ranged from 0 to 4.3 . The amplitude of the saccades was $11.7 \pm 7.4^{\circ}$ (mean $\pm \mathrm{SD}$ ), which ranged from 0.1 to 46.1 . 
A

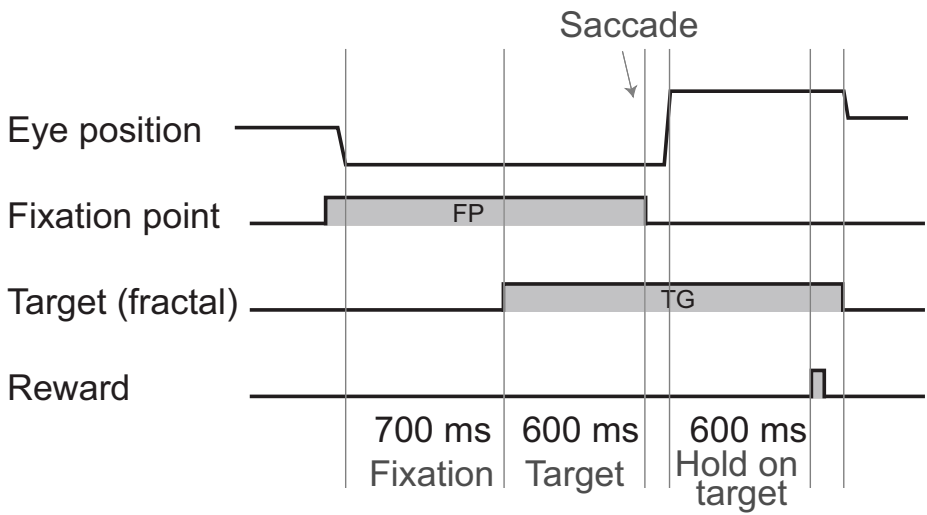

B

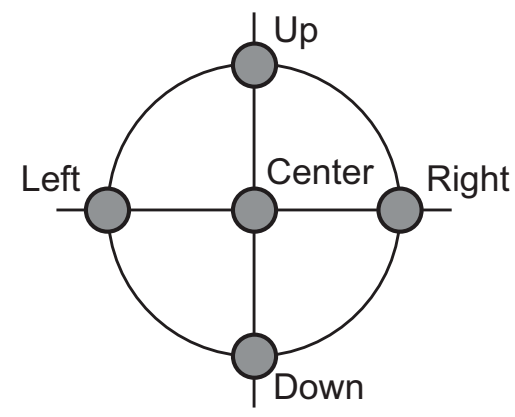

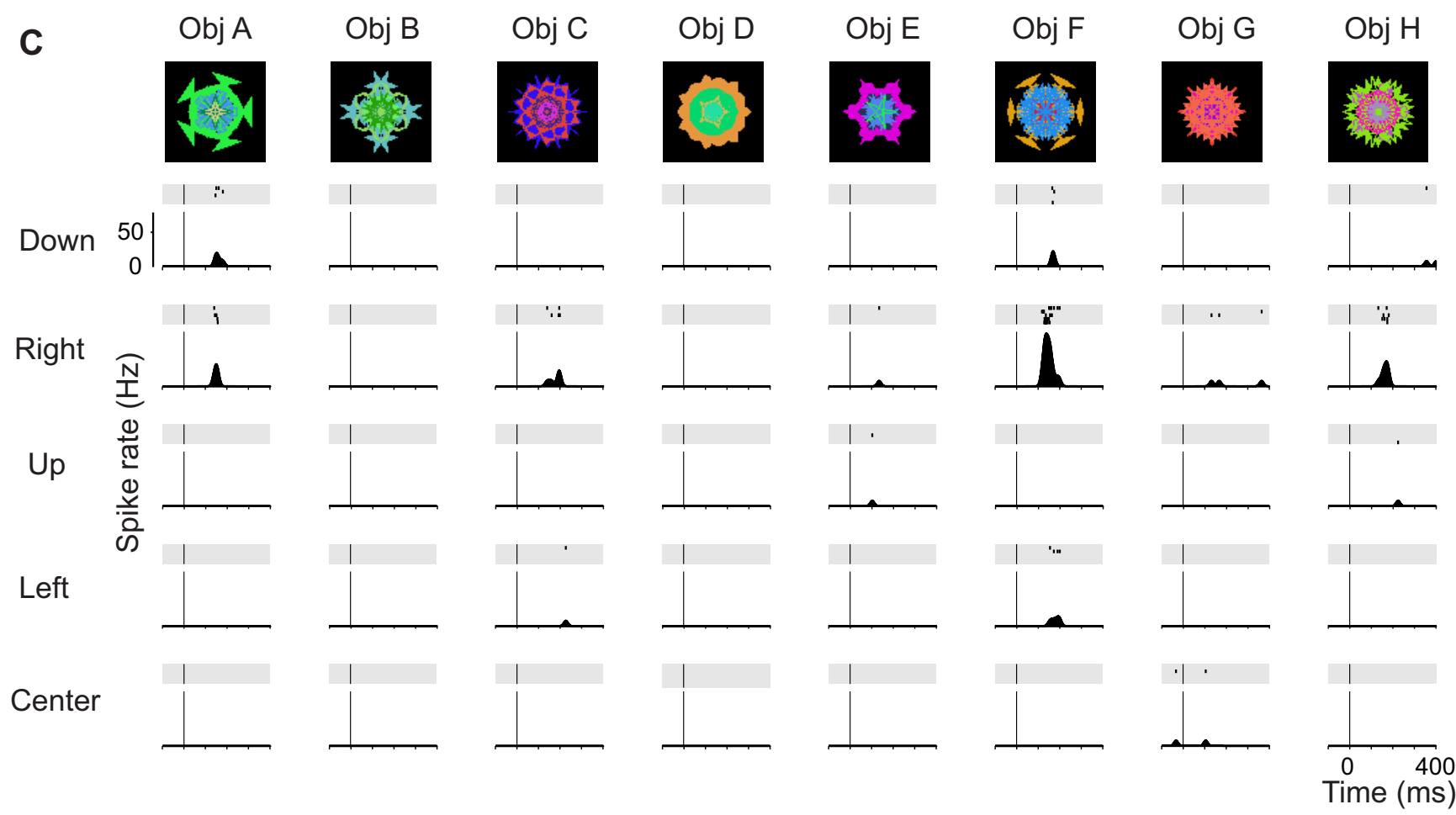

Figure 2. Object and position coding of a (Dt neuron. $\boldsymbol{A}, \boldsymbol{B}$, The object-directed saccade task. While the monkey was fixating on a central spot of light (FP), one of eight fractal objects (TG) (C, top) was presented at one of five positions $(\boldsymbol{B})$. The choice of the target object and position was based on a pseudorandom schedule. The monkey was allowed to make a saccade to the object only after the fixation point disappeared, after which a liquid reward was delivered to the monkey. No saccade was required when the object appeared at the center. C, Responses of a neuron recorded in the left CDt to the appearance of the fractal objects, shown separately for different objects (columns) and different positions (rows). This neuron showed a high selectivity to both object and position information. The spike activity of the CDt neuron is shown by raster displays and spike density functions (SDFs) $(\sigma: 10 \mathrm{~ms})$. The time 0 indicates the onset of the fractal object. For this and other $\mathrm{CDt}$ neurons, we used the same set of fractal objects (standard set) (Figs. 2-5) in addition to some other sets of objects.

To maintain the monkey's arousal level, we included rewarded trials. After every one to four fractal presentations, a small white dot appeared at one of the other four positions $2 \mathrm{~s}$ later, and, if the monkey made saccade to and fixated on it for $3 \mathrm{~s}$, a liquid reward was given to the monkey.

\section{Electrophysiology}

Based on a stereotaxic atlas (Saleem and Logothetis, 2007), a rectangular (28 mm anterior-posterior $\times 26 \mathrm{~mm}$ medial-lateral) or a circular (19$\mathrm{mm}$-diameter) recording chamber was placed over the parietal cortex, tilted laterally by $25^{\circ}$, and aimed at the tail of the CDt. MR images ( $4.7 \mathrm{~T}$; Bruker) were then obtained along the direction of the recording chamber, which was visualized with gadolinium that filled grid holes and inside the chamber.

Single-neuron recordings and electrical stimulations were performed using tungsten electrodes (FHC; diameter, $0.25 \mathrm{~mm}$; 1-3 M $\Omega$; Alpha-Omega; diameter, $0.39 \mathrm{~mm} ; 1-3 \mathrm{M} \Omega$ ). The recording (or stimulation) site was de- termined using a grid system, which allowed electrode penetrations at every $1 \mathrm{~mm}$. Based on the MR images and preceding recording data, we chose a grid hole to hold the stainless-steel guide tube, through which the electrode was inserted and was advanced by an oil-driven micromanipulator (MO97A; Narishige). Based on the grid hole position and the reading of the electrode depth, we estimated the 3D position of the electrode.

The electrical signal from the electrode was amplified with a bandpass filter $(200 \mathrm{~Hz}$ to $10 \mathrm{kHz}$; BAK) and collected at $1 \mathrm{kHz}$ via custom-made window discriminator (MEX). Single neurons were isolated on-line using custom voltage-time window discrimination software (MEX, Laboratory of Sensorimotor Research, National Eye Institute-National Institutes of Health, Bethesda, MD).

To find visually responsive CDt neurons, we let the monkey continue to perform the passive-viewing task or the object-directed saccade task. Since CDt neurons fired spikes only occasionally, we could find and 

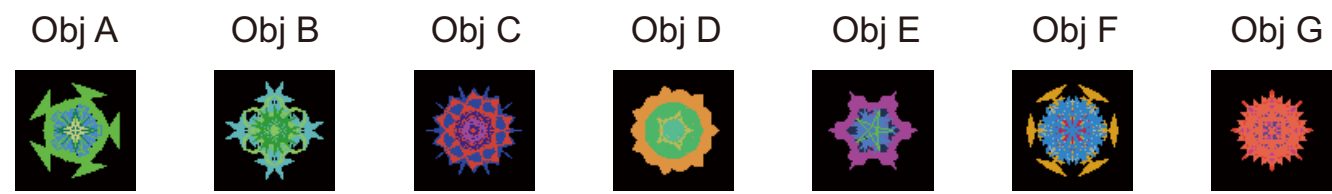

Obj $\mathrm{H}$
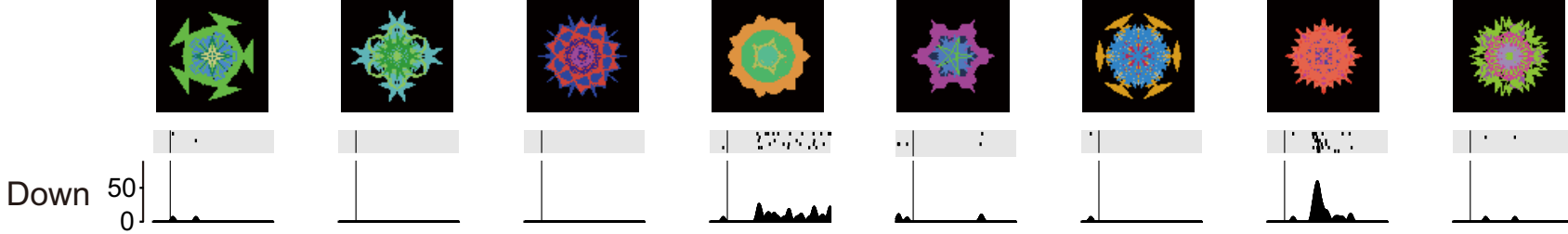

Right
Up
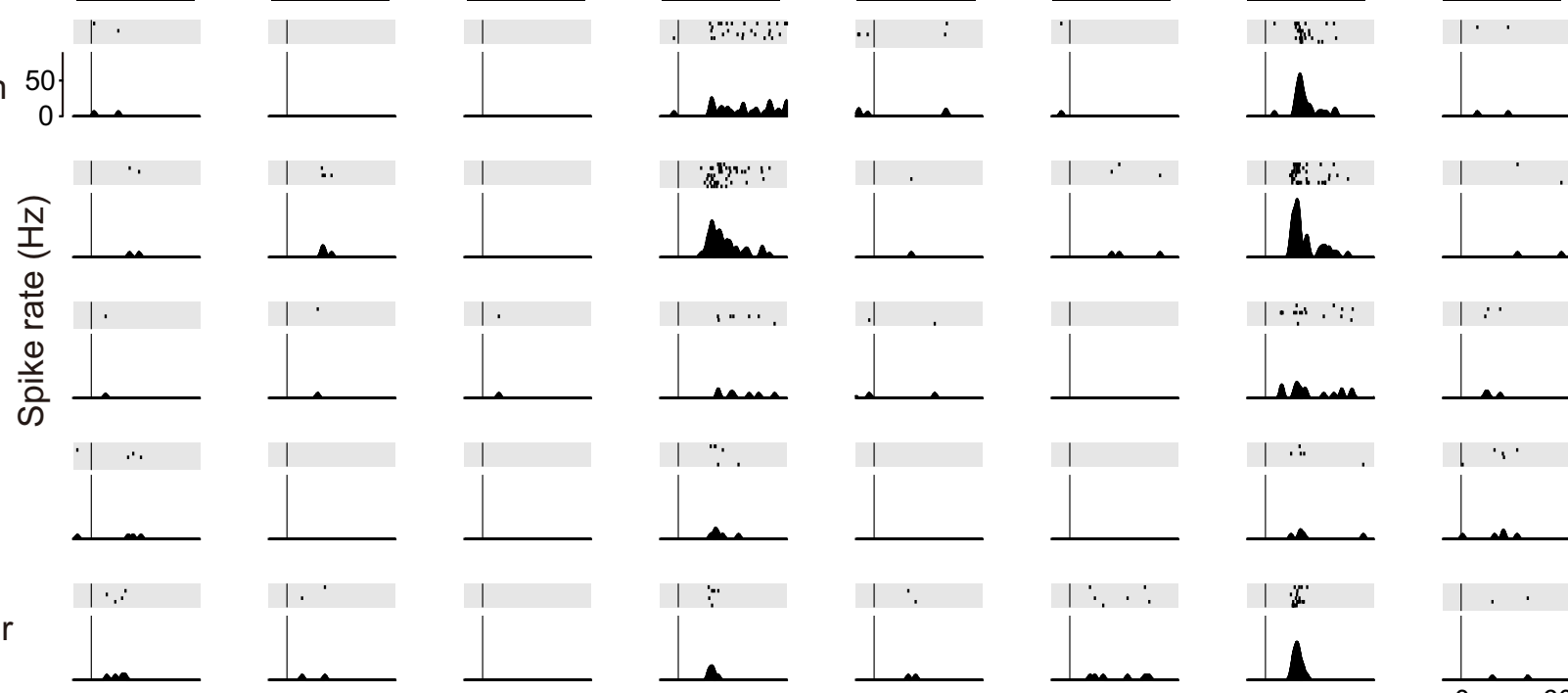

Center
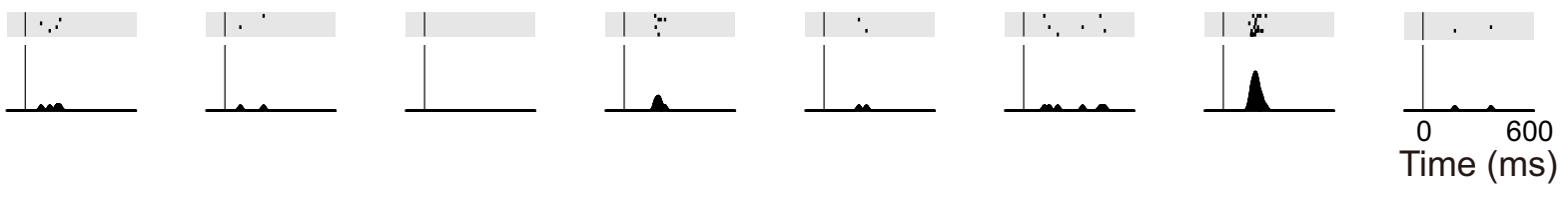

Figure 3. Responses of a neuron recorded in the left CDt. This neuron showed a higher selectivity to object information than to position information. The format is the same as in Figure 2.

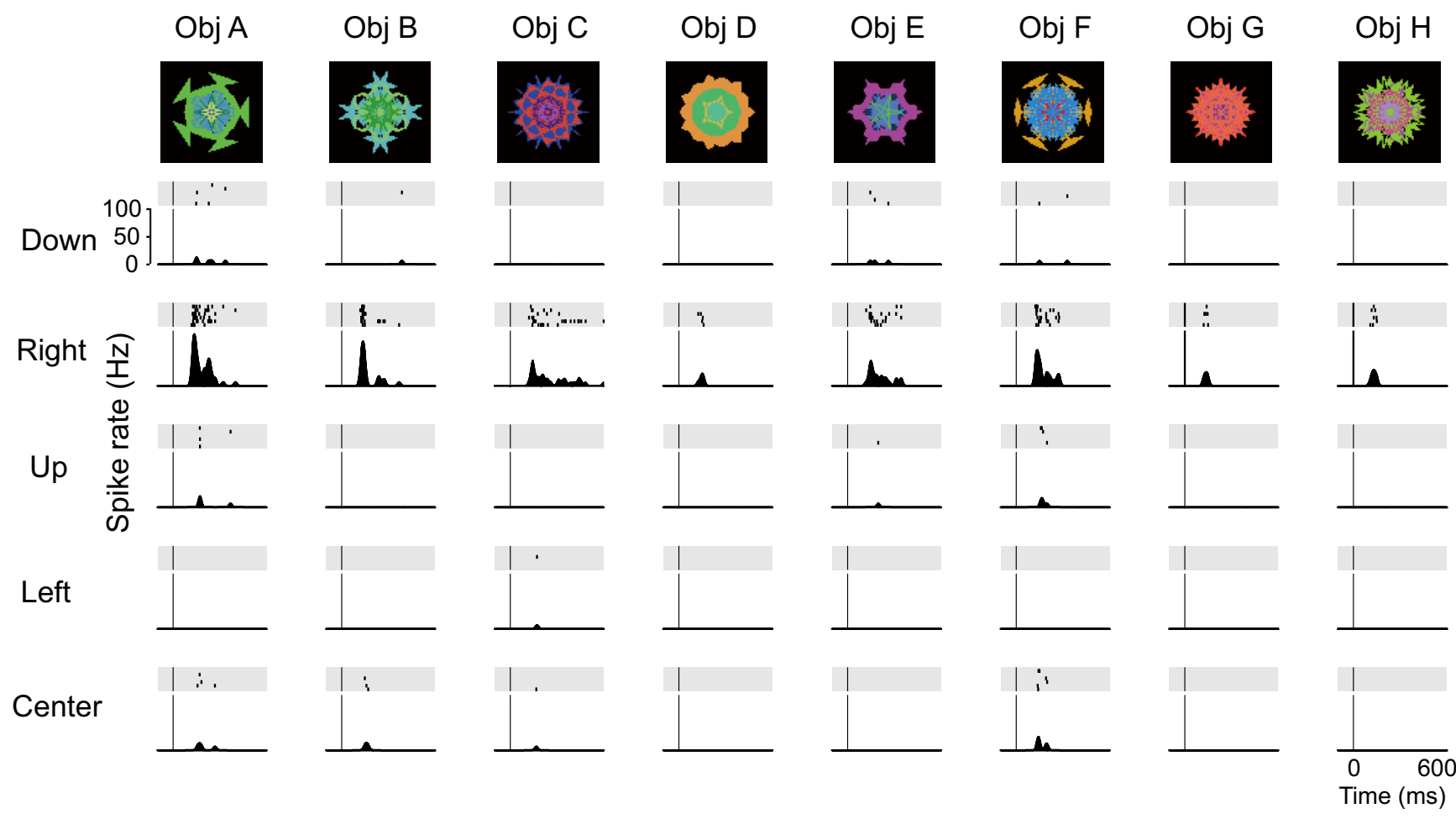

Figure 4. Responses of a neuron recorded in the left CDt. This neuron showed a higher selectivity to position information than to object information. The format is the same as in Figure 2.

examine only those neurons that responded to these visual-saccade tasks. It is thus likely that other nonvisual-saccadic neurons, if present, remained undetected or uncharacterized.

Electrical stimulation

To examine whether CDt neurons could induce saccades, we first identified the CDt by recording single neuronal activity responsive to fractals, and then stimulated the CDt and surrounding areas using the same elec- trode as a monopolar stimulating electrode. We used a train of biphasic negative-positive pulses with $200 \mu$ s per phase duration $(400 \mathrm{~Hz} ; 300$ $\mathrm{ms}$ ) for the stimulation. The amplitude of the electrical currents was 20-60 $\mu \mathrm{A}$. The stimulation experiment was done while the monkey was in a free-viewing condition (fractal presentation time, $2000 \mathrm{~ms}$; blank period, $1000 \mathrm{~ms}$ ). The liquid reward was given to the monkey after one to three presentations of fractals, regardless of the monkey's behavior. Within a subblock 


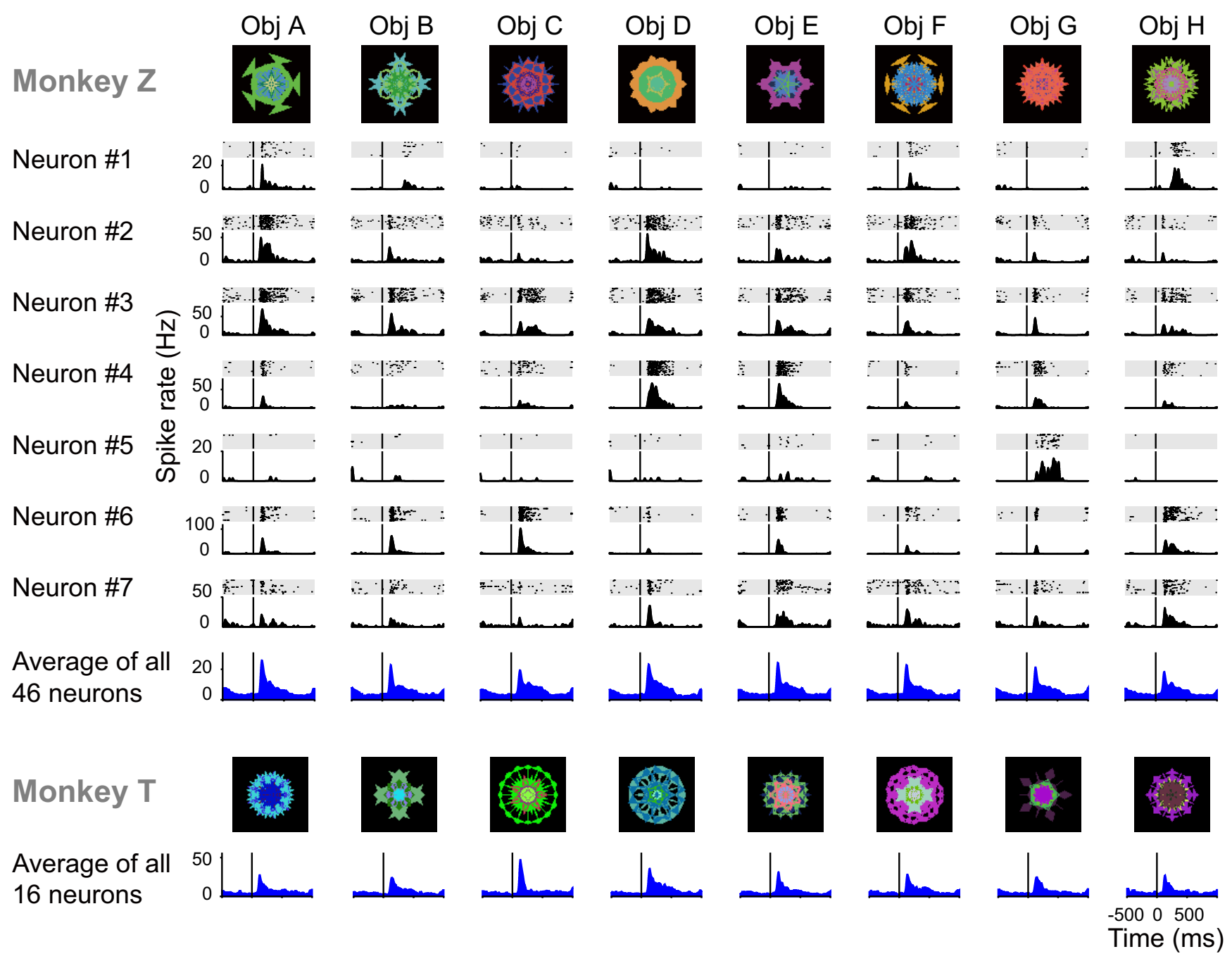

Figure 5. Object selectivity of CDt neurons. The responses of seven CDt neurons to the standard set of fractal objects during the passive-viewing task (see Materials and Methods). While the monkey was fixating on a central spot, two to six objects chosen pseudorandomly from the standard set were presented in sequence at the preferred position of the neuron (presentation time, 400 $\mathrm{ms}$; interpresentation time, $500-700 \mathrm{~ms}$ ). The time 0 indicate the time when the object appeared. Bottom, The average responses of all CDt neurons from monkey $Z$ ( $n=46)$ and monkey $\mathrm{T}(n=$ 16), shown separately for the objects (10 ms $\sigma$ for SDFs). The standard set of objects was different between the two monkeys. Note that the preferred object, response pattern, and latency were different among neurons, but the averaged responses to the objects were similar to each other.

of four trials, two trials were chosen randomly for electrical stimulation, one for stimulation during the fractal presentation period and the other for stimulation during the blank period. An electrical stimulation was applied at a random timing during the chosen period. These procedures were used to minimize the variation of the monkey's arousal, cognitive, and emotional states.

\section{Data analysis}

Object and position selectivity of the visual responses of CDt neurons. For each neuron that responded to fractal objects, we examined whether its response was object and/or position selective. For this purpose, we conducted a two-way ANOVA (5 positions $\times 8$ objects) for neuronal activities during the objectdirected saccade task. The number of spikes during the FP-TG overlap period (while the fractal object was present and while the monkey was fixating the central fixation point) was used as a dependent variable (Table 1).

Object selectivity. The two-way ANOVA does not indicate which objects are preferred and how strongly they are preferred. To answer this question, we performed a receiver operating characteristic (ROC) analysis comparing the response of the neuron to one object with its responses to the other objects. The responses for the best position of the neuron were used for this analysis. Since we used the same standard set of eight fractal objects for 86 neurons (65 neurons in monkey $\mathrm{Z}$ and 21 neurons in monkey $\mathrm{T}$ ), the analysis yielded, for each neuron, eight values corresponding to the area under the ROC curve. The vector of these values is color-mapped for each neuron (see Fig. 6, vertical column).

To quantify how object selective each neuron was, we defined a selectivity index (SI). The SI was calculated for each neuron using the averaged responses to the most and least preferred objects among the eight objects: $\mathrm{SI}=(\max -$ $\min ) /(\max +\min )($ Sereno and Maunsell, 1998; Sereno and Lehky, 2011).

In addition to the object selectivity for individual neurons, we asked whether CDt neurons, as a population, responded to the standard objects differentially. To answer this question, we conducted a Friedman's test for the data of the passive-viewing task ( 8 objects $\times 46$ neurons).

Position selectivity. To examine the preferred positions of CDt neurons, we used two methods: one based on the object-directed saccade task and the other based on the passive-viewing task.

Using the object-directed saccade task, we calculated the "center of gravity" of visual responses based on the number of spikes during the FP-TG overlap period using the following equation:

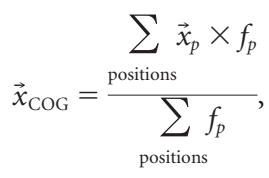




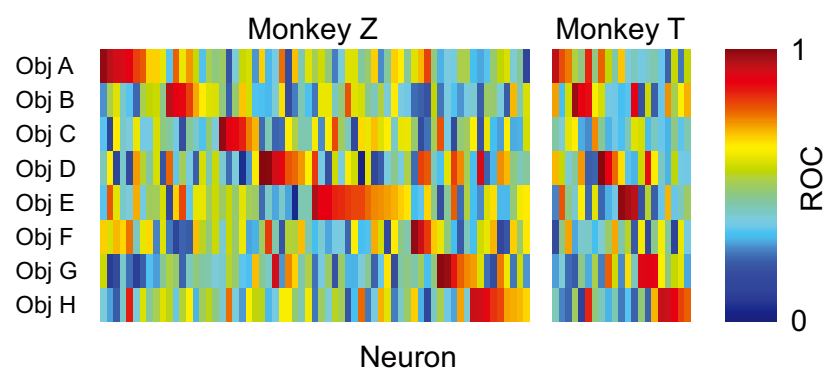

Figure 6. Distributed representation of objects among CDt neurons. The responses of CDt neurons from monkey $Z$ ( $n=65$ ) and monkey $T(n=21)$ to the standard set of eight objects $(\mathrm{A}-\mathrm{H})$ are color-coded across neurons (horizontal axis). For each neuron (vertical column), we normalized its responses to the eight objects (red, strong; blue, weak). For the normalization, we calculated nonparametric ROC areas by comparing the magnitude of the response to each of the eight objects with the responses to the other seven objects. The data were obtained using the object-directed saccade task. Note that each object was most preferred by some of CDt neurons.
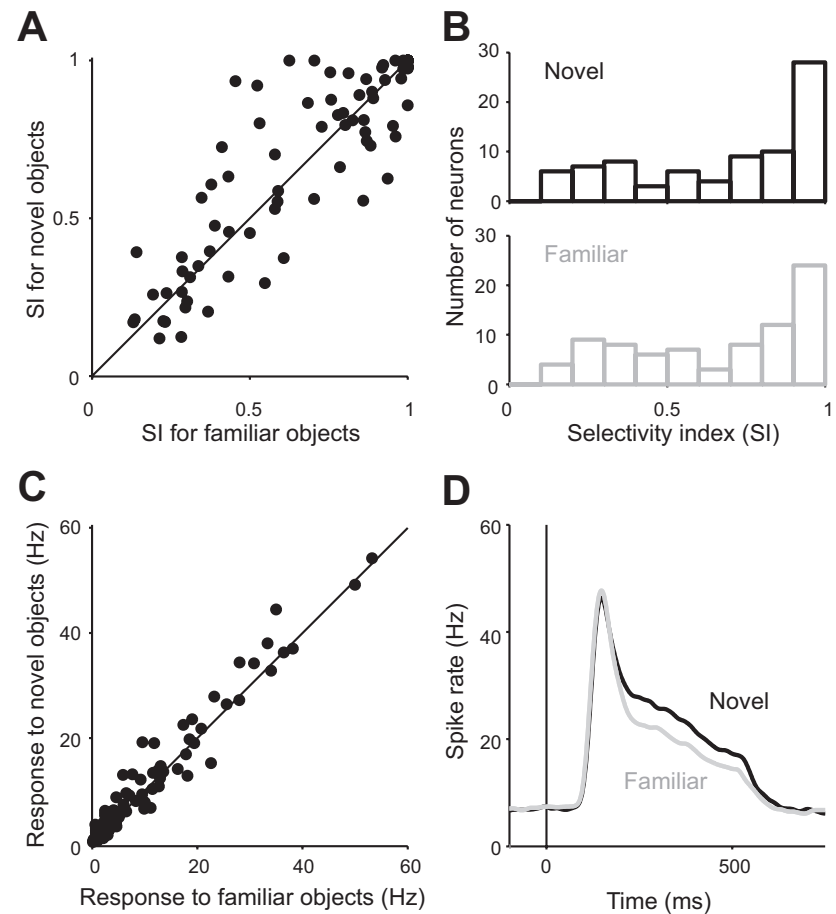

Figure 7. Object selectivity for novel and familiar stimuli of CDt neurons. $A$, SIs for novel and familiar objects are plotted for individual CDt neurons. For each neuron, its responses were examined separately for familiar objects (i.e., the standard set of 8 stimuli) and for novel objects (a set of 8 newly created fractal objects). The data were obtained from 80 neurons using 2 familiar sets (1 for each monkey) and 80 novel sets of fractal objects. $\boldsymbol{B}$, Distributions of SIs for novel objects (top) and familiar objects (bottom). C, Response magnitudes for novel and familiar objects are plotted for individual CDt neurons. $\boldsymbol{D}$, The average responses for novel and familiar objects of the $80 \mathrm{CDt}$ neurons.

where $\vec{x}_{\mathrm{COG}}, f_{p}$, and $\vec{x}_{p}$ denote the center of gravity, the averaged firing rate for each position, and the vector representation of each position $[(15,0)$, $(0,15),(-15,0),(0,-15),(0,0)$ for the right, up, left, down, and center, respectively] (see Fig. $8 \mathrm{~A}$ ). We used the most preferred object of the neuron for this calculation. This method does not take eccentricity into account but indicates whether the neuron preferred peripheral positions or central positions. We also performed an ROC analysis comparing the response of the neuron to one position with its response to the other four positions (see Fig. 8 B).

Using the passive-viewing task, we determined the preferred position by presenting a preferred object at different positions while changing

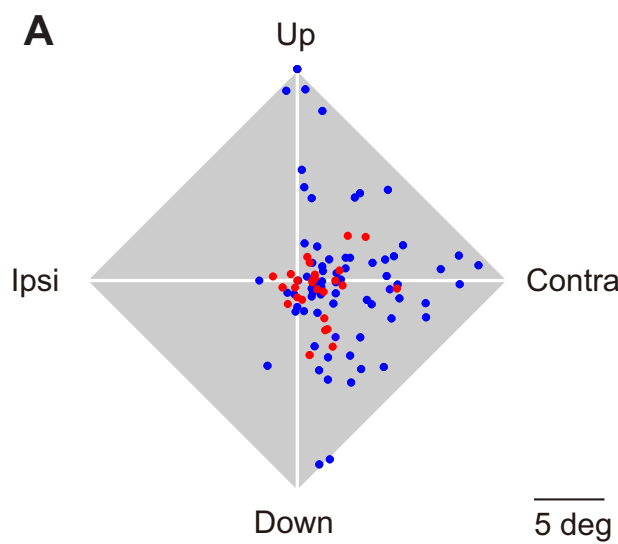

B

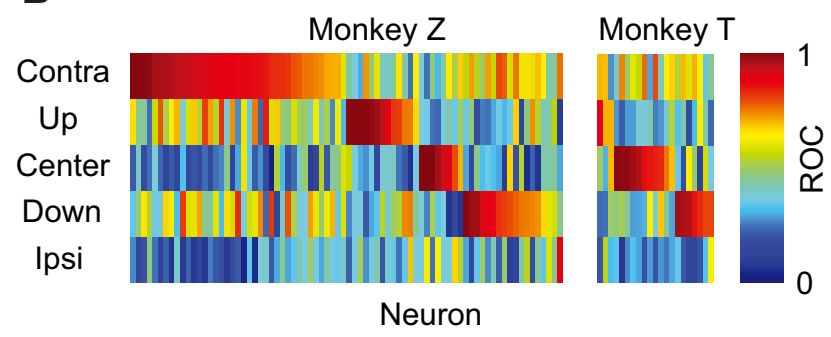

Figure 8. Position selectivity of CDt neurons. $A$, Preferred positions of all CDt neurons. For each neuron, the center of gravity was computed based on the visual responses to the five positions (Fig. 2B) (see Materials and Methods) and is indicated by a dot. The neurons were classified into two types depending on whether their visual responses at the center of gaze were larger (red) or smaller (blue) than the average of the responses to the other four locations. Note that the preferred positions are largely localized in the contralateral hemifield. $\boldsymbol{B}$, The responses of the CDt neurons to five positions were normalized and are color-coded across neurons (horizontal axis) for the two monkeys. The normalized responses were obtained using ROC areas, which were calculated by comparing the magnitude of the response to each of the five positions with the responses to the other four positions. The data in $\boldsymbol{A}$ and $\boldsymbol{B}$ were obtained using the object-directed saccade task.

both direction and eccentricity. The results provide a rough profile of the receptive field of the neuron.

Saccades induced by electrical stimulation of the CDt. Since the monkey made saccades spontaneously while the electrical stimulation was applied occasionally, we needed to differentiate between the spontaneous saccades and the stimulation-induced saccades. To this end, we compared the saccades that occurred during the electrical stimulation and the saccades that occurred during an equivalent behavioral state but with no electrical stimulation.

For each electrical stimulation, we detected the saccade that occurred first after the electrical stimulation started (but within $500 \mathrm{~ms}$ ) as a candidate of a stimulation-induced saccade. The distribution of the vectors of the first saccades (see Fig. 10 A) was smoothed with a two-dimensional Gaussian kernel $\left(\sigma=5^{\circ}\right)$. We then defined a "movement field" as an area whose values were $>60 \%$ of the peak value (after subtracting the background value).

Free-viewing condition. We hypothesized that the object-selective responses of CDt neurons can induce saccades to particular objects when the monkey explore the visual environment regardless of reward outcome. To test the hypothesis, we analyzed the activity of CDt neurons in the free-viewing condition in relation to such spontaneous saccades. Among all saccades that occurred in the free-viewing condition, we concentrated on (1) the saccades directed to the most preferred object of the neuron (among the eight objects) and (2) the saccades directed to the least preferred object. We determined the most and least preferred objects using the passive-viewing task. Each group of saccades was further divided into two groups: (1) saccades directed to the most preferred direction of the neuron and (2) saccades directed to the least preferred direction. We defined the most preferred direction to be within $\pm 90^{\circ}$ of 


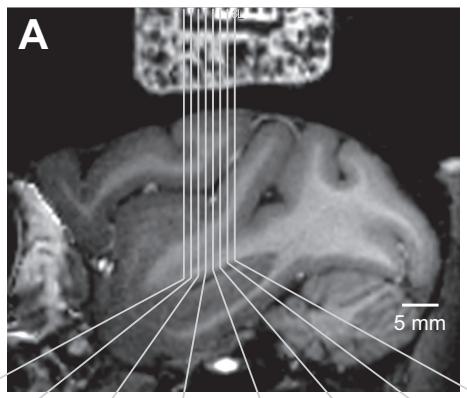

B

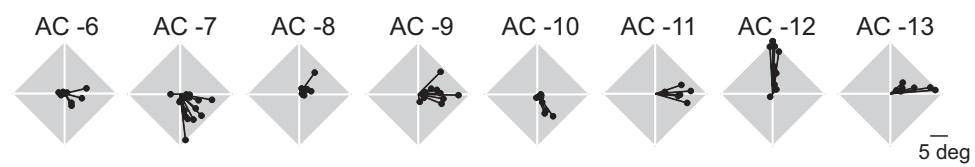

Figure 9. Variation of position selectivity $(\boldsymbol{B})$ along the anterior-posterior axis of the $\operatorname{CDt}(\boldsymbol{A}) \cdot \ln \boldsymbol{B}$, the preferred positions are shown at each anterior-posterior location indicated in the MR image in $A$. Data were obtained from one monkey (monkey $Z$ ). The anterior-posterior location was measured from the position of the AC. For example, "AC -6 " indicates " $6 \mathrm{~mm}$ posterior from the $A C$." The preferred positions were computed as the centers of gravities for individual neurons. The MR image was aligned with the electrode tracks (as in Fig. 1A); the rectangular object at top is the recording chamber and reference grid (both filled with gadolinium to allow MRI visualization).
A

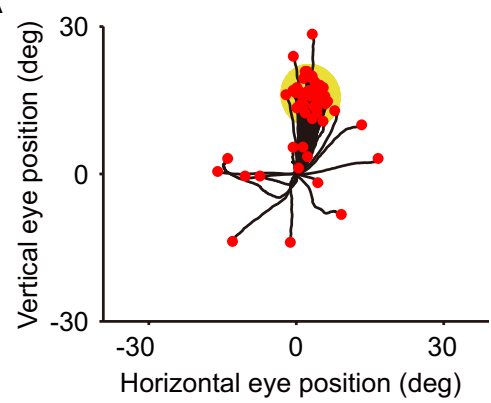

C

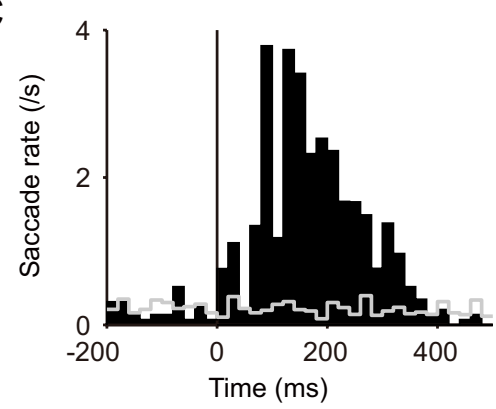

B

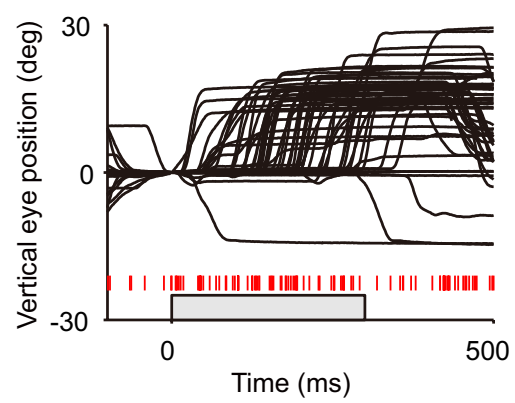

D

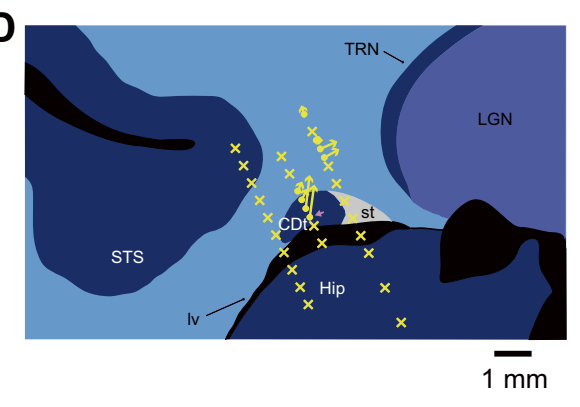

Figure 10. Saccades induced by electrical stimulation of the CDt. $\boldsymbol{A}, \boldsymbol{B}$, Saccades during stimulation $(40 \mu \mathrm{A})$ at a point in the $C D t$, which is indicated by a pink arrow in $\boldsymbol{D}$. $\boldsymbol{A}$, The trajectories of the first saccades within $500 \mathrm{~ms}$ after electrical stimulation started. The endpoints of the saccades are shown by red dots, and a movement field (see Materials and Methods) is indicated by a yellow area. $\boldsymbol{B}$, Superimposed vertical eye positions aligned on the electrical stimulation (gray rectangle). The onsets of all saccades are indicated by a raster of short red lines. In $\boldsymbol{A}$ and $\boldsymbol{B}$, the eye positions (expressed in degrees) have been adjusted to zero at the beginning of the stimulation. $\boldsymbol{C}$, The change in the probability of saccades into the movement field relative to stimulation onset (black histogram). The saccades from multiple locations in the CDt ( $n=$ 12) are included (Fig. 11). A gray line indicates the change in the probability of saccade into the movement field relative to pseudostimulation onset (i.e., time at which electrical stimulation would have occurred but did not). The data in $\boldsymbol{A}-\boldsymbol{C}$ were obtained using the currents of $40 \mu \mathrm{A}$. D, Localization of the stimulation effects. In this coronal plane, electrical stimulation was delivered along three adjacent electrode tracks. The vector (direction and amplitude) to the peak of the movement field is shown by a yellow arrow whose starting point indicates the stimulation site. The crosses indicate the sites from which no saccades were induced with the currents of $20 \mu \mathrm{A}$. the direction of the center of the receptive field and the least preferred direction to be within $\pm 90^{\circ}$ of the opposite to the center or the receptive field. We then analyzed CDt activity separately for the four groups: most preferred and least preferred (object by position) (see Figs. $12,13)$. To analyze the population data, we performed a three-way ANOVA $(2$ objects $\times 2$ directions $\times 66$ neurons) for the period of 200 ms before the saccade.

\section{Histology}

In the later part of the experiments in monkey $\mathrm{T}$, we made electrolytic microlesions at the recording and stimulation sites $(12 \mu \mathrm{A} ; 30 \mathrm{~s})$. We chose several sites for the microlesions along the anterior-posterior axis of the CDt. For each site, we made two to three microlesions with different patterns of intervals, one of them usually inside the CDt. The animal was then deeply anesthetized with pentobarbital sodium and perfused with $4 \%$ paraformaldehyde. Frozen sections were cut every $50 \mu \mathrm{m}$ in the coronal plane. The sections were stained with cresyl violet (see Fig. 1C,D).

\section{Results}

Encoding of what and where information in the $\mathrm{CDt}$

We recorded spike activity of single neurons in a wide area in the CDt in two monkeys while they viewed and made saccades to fractal objects. We confirmed that the locations of our neuronal recordings were confined to the CDt in three ways. First, we physiologically identified nearby structures, particularly the lateral geniculate nucleus (Malpeli and Baker, 1975) (Fig. $1 C, D)$, to guide the position of the recording electrode. Second, we observed neuronal spike shapes and firing patterns typically observed within the primate caudate nucleus (Hikosaka et al., 1989). Third, we obtained definitive evidence of our recording location by the histological examination of electrolytic microlesions, which were made at the end of the recording experiments (Fig. 1D).

We found that many neurons in the CDt responded to fractal objects, although it was unclear how prevalent such object-responsive neurons were in the CDt (see Materials and Methods). To examine the object and position selectivity of CDt neurons, we used an objectdirected saccade task (Fig. 2 A). The target object was chosen from a standard set of eight fractals (Fig. 2C, top). On each trial, one of the eight fractal objects was presented at one of five positions (Fig. $2 B$ ), and the monkey made a saccade to it to obtain a fixed amount of reward (water or juice).

A majority of the object-responsive CDt neurons responded to the fractal ob- 
jects in an object-selective and positionselective manner. Spike activity of an example neuron recorded in the left $\mathrm{CDt}$ is shown in Figure $2 C$. This neuron rarely fired spikes spontaneously and thus was considered to be a medium-spiny projection neuron (Plenz and Kitai, 1998). It responded with a brief excitation to some objects (e.g., Obj F), particularly when they were presented in the rightward direction (which was contralateral to the recording site) (Fig. 2C). Other examples are shown in Figures 3 and 4.

A two-way ANOVA (object by position) revealed that, among $99 \mathrm{CDt}$ neurons that responded to fractal presentation, 76 neurons (77\%; including neurons in Figs. 2C, 3, 4) showed significant main effects of both object and position $(p<0.05), 97$ neurons $(98 \%)$ showed main significant effects of either object or position $(p<0.05)$, and 63 neurons $(67 \%)$ showed significant effect of the interaction of object and position $(p<0.05$; Table 1). Thus, the CDt encodes both what and where information about visual objects.

The object selectivity varied across CDt neurons (Fig. 5). Different neurons preferred different objects, and even the responses of one neuron had different time courses across the objects (Fig. 5, compare neurons 1, 7). Overall, the averaged response across all neurons examined was similar across the objects (Fig. 5, bottom) ( $p=0.66$ for monkey $\mathrm{Z}$ and $p=$ 0.77 for monkey T, Friedman's test). Conversely, each object was most preferred by a fraction of CDt neurons (Fig. 6, indicated by red) and least preferred by a different fraction of CDt neurons (Fig. 6, blue). These results suggest that, on average, the population of $\mathrm{CDt}$ represented all of the eight objects comparably well.

Note that both monkeys had seen the eight objects many times during training and recording sessions. To test whether the object preference of $\mathrm{CDt}$ neurons was acquired through experience, we created novel fractal objects and used them as additional visual stimuli in the same task. We found that their responses to the novel objects were object selective similarly to the familiar objects (Fig. $7 A, B$ ). There was no significant difference in the mean SI between novel objects (mean, 0.69) and familiar objects (mean, $0.67)$ ( $p=0.31$, paired $t$ test). These results suggest that the object-selective responses of CDt neurons do not require experience. Note, however, that the magnitude of response was overall stronger for novel stimuli than for familiar stimuli $(p=2.8 \times$ $10^{-3}$, paired $t$ test) (Fig. $7 C, D$ ).

The position selectivity also varied across CDt neurons, but their preferred directions were largely confined to the hemifield contralateral to the recording sites (Fig. 8). To describe the spatial properties of CDt neurons, we computed the center of gravity, which is a directional vector based on the response magnitudes for the five positions (Fig. 2B) (see Materials and Methods). The data points close to the center correspond to neurons that responded to the center more strongly than the peripheral posi-
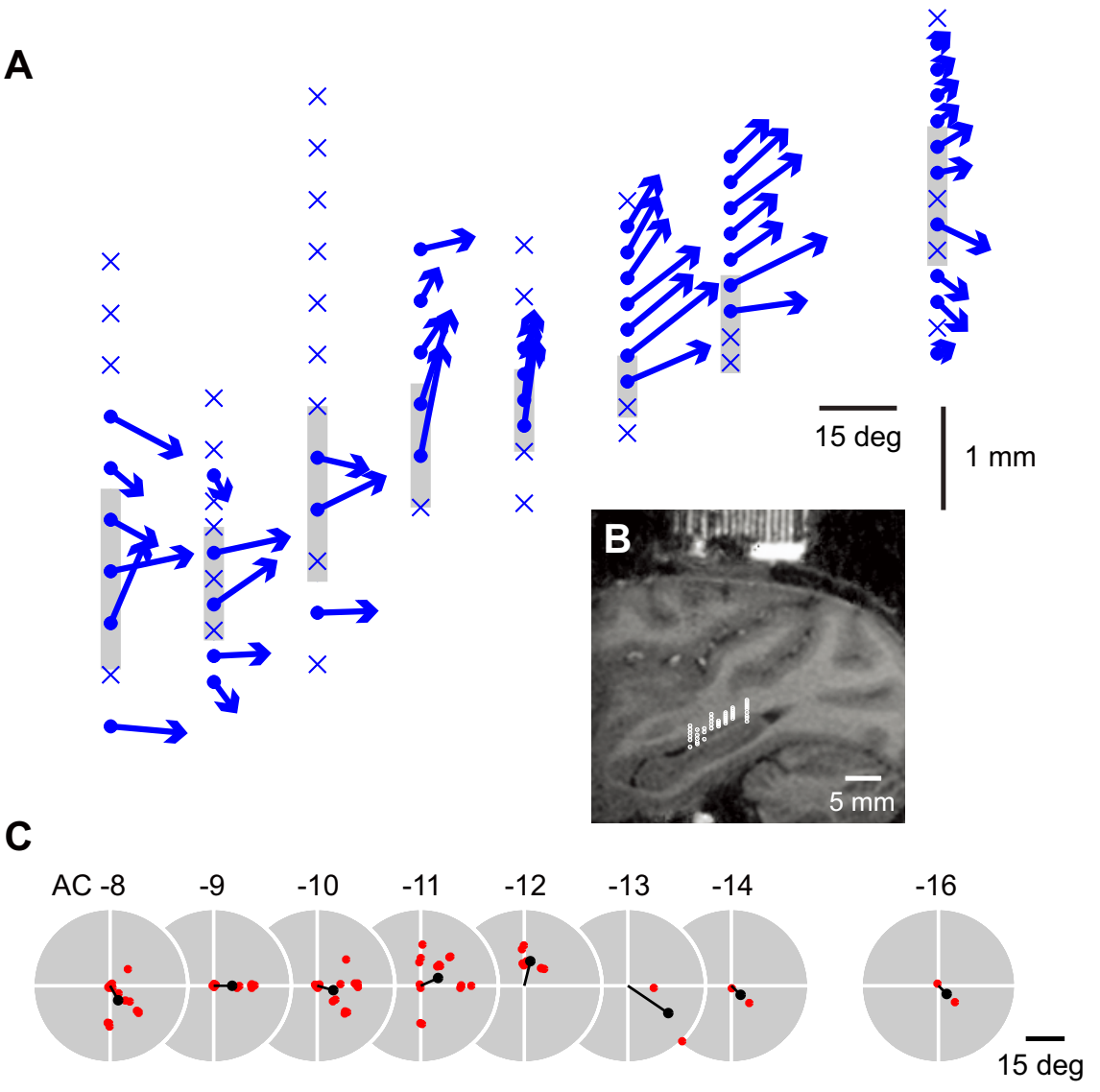

Figure 11. Variation of stimulation-induced saccades along the anterior-posterior axis of the CDt. $\boldsymbol{A}$, The vector to the peak of the movement field is plotted at each stimulation site. The crosses indicate the sites from which no saccades were induced. The gray positions of the visual responses of CDt neurons at the anterior-posterior locations that correspond to the data in $\boldsymbol{A}$. The preferred positions are shown for individual neurons (red dots) and their average (black dots). The anterior-posterior positions are measured from the anterior commissure (AC). Data were obtained from one monkey (monkey T).

tions. The horizontal coordinate of the center of gravity was significantly deviated to contralateral side (mean $\pm \mathrm{SD}, 2.73 \pm$ 3.04; $p=2.6 \times 10^{-14}, t$ test), but the vertical coordinate was not significantly deviated (mean $\pm \mathrm{SD},-0.02 \pm 4.65 ; p=0.97, t$ test).

In Figure $8 \mathrm{~A}$, we divided CDt neurons into two groups by comparing their responses to objects at the center with the other four positions in the object-directed saccade task: neurons that responded to the central position more strongly than to the average of four peripheral positions (red dots) and neurons that responded to the central position less strongly than to the average of four peripheral positions (blue dots).

We found that the representation of space is organized within the CDt. In Figure 9B, the centers of gravity of CDt neurons (as shown in Fig. 8A) are shown separately for different anteriorposterior levels of recording, which are indicated on an MRI image (Fig. 9A). For example, the center of gravity was mostly upward at the position of $\mathrm{AC}-12[12 \mathrm{~mm}$ posterior from the anterior commissure $(\mathrm{AC})$ ], contralateral at $\mathrm{AC}-9$, and downward at $\mathrm{AC}-7$. However, the same directions appear represented more than once along the anterior-posterior axis, and therefore the spatial organization in the CDt is not a simple topographic map as seen in the primary visual cortex or the superior colliculus. Nonetheless, the CDt is different from other brain regions with sharp object selectivity, such as the anterior inferotemporal 
cortex, which display no clear spatial organization (Tanaka, 1996). Thus, the CDt seems uniquely suited for controlling motor behavior aiming at visual objects.

\section{Electrical stimulation in the CDt induces saccades}

The output of the caudate nucleus, including that of the CDt, is directed exclusively to other basal ganglia structures, specifically the substantia nigra and the globus pallidus (Hikosaka et al., 2000). In particular, the efferent connection of the CDt to the dorsal part of the $\mathrm{SNr}$ (Saint-Cyr et al., 1990) suggests that the $\mathrm{CDt}$ can modulate the initiation of saccadic eye movements, because this part of the $\mathrm{SNr}$ projects to the SC (Beckstead et al., 1981) and has been demonstrated to play a role in the control of saccadic eye movements (Hikosaka and Wurtz, 1983b).

We found indeed that electrical stimulation of the CDt induced saccades (Fig. 10). We stimulated the $\operatorname{CDt}(20-60 \mu \mathrm{A})$ at random intervals while the monkey was free viewing fractal objects (see Materials and Methods). We first determined that the electrode was within the CDt by recording from several neurons showing object- and position-selective spike activity, which was later confirmed histologically (Fig. 1D). At this stimulation site, the probability of saccades increased during the electrical stimulation (Fig. $10 B$ ) and the saccades were largely directed upward (Fig. 10A). Based on the distribution of saccade endpoints, we defined a movement field at this CDt site (Fig. $10 \mathrm{~A}$, yellow area). The probability of saccades to the movement field was elevated just after the electrical stimulation started, peaked at $\sim 100 \mathrm{~ms}$, and then gradually decreased to the baseline until the end of electrical stimulation (Fig. 10C, black histogram). When there was no electrical stimulation, there was no increase in the probability of saccades to the movement field (Fig. 10C, gray line). These results indicate that the saccades to the movement field were caused or facilitated by electrical stimulation of the CDt. Hereafter, we call them stimulation-induced saccades.

We stimulated different sites in and around the CDt and found similar results (Fig. 10D). Other sites near the CDt were mostly ineffective, even when they were $<1 \mathrm{~mm}$ medial, lateral, or ventral to the $\mathrm{CDt}$ (Fig. 10D). We occasionally found effective patchy regions outside the $\mathrm{CDt}$ (Fig. $10 D$, the right track), which could be due to activation of a bundle of axons afferent to the $\mathrm{CDt}$ or efferent from the CDt.

The stimulation-induced saccades varied depending on the anterior-posterior position of the $\mathrm{CDt}(\mathrm{Fig} .11 \mathrm{~A})$. At the site 12 $\mathrm{mm}$ posterior to the anterior commissure $(\mathrm{AC}-12)$, the directions of stimulation-induced saccades were upward (as shown in Fig. 10). Moving anteriorly from AC -12 , the saccade direction rotated clockwise to rightward (i.e., contralateral) and slightly downward. The directions of stimulation-induced saccades roughly correlate with the preferred positions of visual responses of CDt neurons recorded at the stimulation sites (Fig. 11C) (see Materials and Methods). These data suggest that the visual object information in the CDt could be transformed into saccadic motor information in a spatially heterogeneous manner.

Moving posteriorly from $\mathrm{AC}-12$, the amplitude, rather than direction, changed from large $(\mathrm{AC}-13)$ to small $(\mathrm{AC}-16)$. In these posterior regions, the effective sites covered $1-2 \mathrm{~mm}$ above the $\mathrm{CDt}$ in addition to the $\mathrm{CDt}$ itself, but the stimulation-induced saccades tended to be larger from the sites in the CDt. Unlike the anterior regions, the directions of the stimulation-induced saccades were different from the preferred positions of the CDt neurons (mostly right-downward; Fig. 11C).
A

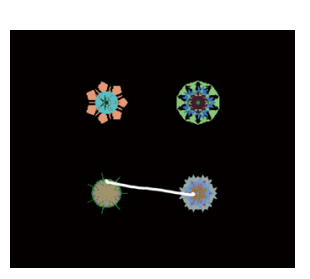

B
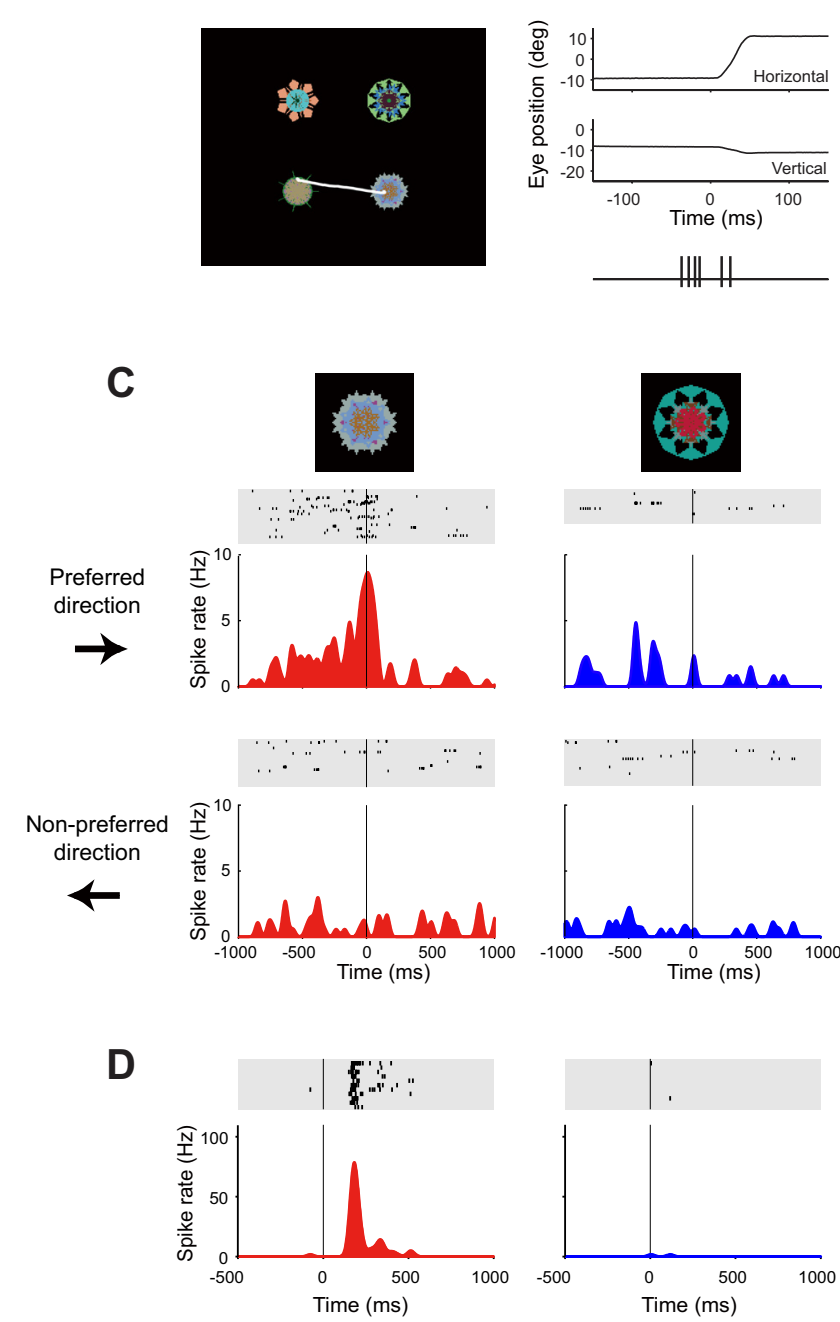

Figure 12. Comparison between presaccadic activation in the free-viewing condition and visual responses in the passive-viewing task in a CDt neuron. $\boldsymbol{A}$, Simultaneously presented fractal objects which the monkey was allowed to look at freely with no reward outcome, while activity of one CDt neuron was being recorded. The white line indicates the trajectory of a saccade directed to the right, which is also shown in $B$ as changes in horizontal (top) and vertical (bottom) eye positions. At bottom of $\boldsymbol{B}$ are shown spikes of the CDt neuron that occurred before the saccade. $\boldsymbol{C}$, The spike activity of the neuron aligned on saccades directed to the most preferred (left column) and the least preferred (right column) objects of the neuron, separately for the preferred (top row) and the nonpreferred (bottom row) directions of the neuron ( $20 \mathrm{~ms} \sigma$ for SDFs). The time 0 indicates the time when the saccades started. Since the preferred direction was horizontally rightward (contralateral), the saccades collected in the free-viewing condition were grouped into "preferred" if their directions were within $\pm 90^{\circ}$ from the horizontal rightward and "nonpreferred" if their directions were within $\pm 90^{\circ}$ from the horizontal leftward. $\boldsymbol{D}$, The responses of the neuron to the most preferred object (left) and least preferred object (right) in the passive-viewing task. The objects were presented at the preferred position of the neuron, rightward. The time 0 indicates the time when the object appeared. The object disappeared at $400 \mathrm{~ms}$.

\section{Neuronal activity during free viewing}

These data suggest that the CDt has a mechanism to influence saccades but do not indicate that the CDt actually transmits signals before the initiation of saccades. Since the visual responses of CDt neurons are object selective, the CDt neurons may contribute to the initiation of saccades only when the saccades are directed to the preferred objects of the neuron. To test this hypothesis, we first determined the object and position preference of a CDt neuron using the passive-viewing task and the 
responses were rank-ordered to determine the most- and least-preferred objects (Fig. 12D) (see Materials and Methods). We then recorded the activity of the neuron while the monkey was freely looking at multiple objects (Fig. 12A-C). In the free-viewing condition, four fractal objects were chosen randomly from a set of eight objects and were presented simultaneously for $3 \mathrm{~s}$ (Fig. $12 \mathrm{~A}$ ). Even though no reward was given after the object presentation, the monkey typically looked at these objects by making saccades between objects several times in the 3 s period. An example saccade is shown in Figure 12, $A$ and $B$ (top), together with spike activity of a CDt neuron (Fig. $12 B$, bottom).

In Figure $12 C$, the activity of the $\mathrm{CDt}$ neuron is aligned on saccades that were directed to the most preferred (left) and the least preferred (right) objects of the neuron. In each case, the activity of the neuron was further divided into two groups based on the directions of the saccades: the preferred (top) and the nonpreferred (bottom) directions of the neuron. The neuron became active before saccades only when they were directed to the preferred object and when the saccades occurred in the preferred direction (Fig. $12 C$, top left).

A similar object direction selectivity was commonly observed in CDt neurons, as indicated by the population data (collected from 66 CDt neurons) (Fig. 13). To see the time course of the presaccadic activity more clearly, we collected saccades that were not preceded by another saccade within $500 \mathrm{~ms}$. As shown in Figure $13 B$, the average spike activity grew larger when saccades were directed to the preferred object in the preferred direction compared with the other saccades in $<200 \mathrm{~ms}$ before the saccade onset (Fig. 13B). These data are consistent with the hypothesis that individual CDt neurons contributes to the initiation of saccades directed to specific objects located in specific positions.

\section{Discussion}

Our experiments have shown that many neurons in the monkey CDt respond to fractal pictures in object- and position-selective manners. The object selectivity is expected from anatomical studies showing that the cortical areas in the ventral visual pathway project to the CDt (Yeterian and Van Hoesen, 1978; Van Hoesen et al., 1981; Saint-Cyr et al., 1990; Webster et al., 1995) and is consistent with two previous single-unit recording studies (Caan et al., 1984; Brown et al., 1995). However, the position selectivity in CDt neurons is unexpected, since position selectivity tends to become weaker along the ventral visual pathway (Boussaoud et al., 1991). One explanation would be that object-selective neurons in the inferotemporal cortex (ITC) may carry position information (Op De Beeck and Vogels, 2000; DiCarlo and Maunsell, 2003; Lehky et al., 2008; Sereno and Lehky, 2011). In addition to receiving inputs from the anterior ITC, individual CDt neurons may receive inputs from multiple cortical areas along the ventral visual pathway, including the areas in its earlier stages, such as the central and posterior ITCs, and area V4, which retain some positional information. This is supported by ana- tomical data (Yeterian and Van Hoesen, 1978). A majority of neurons in the caudate nucleus are medium spiny neurons that have hyperpolarized resting potentials (Plenz and Kitai, 1998) and therefore are unlikely to be activated by these inputs individually. Consistent with this view, many of the CDt neurons we recorded rarely fired spikes spontaneously (Figs. 2C, 3, 4). According to this scheme, the object-selective inputs from the anterior ITC could activate CDt neurons only when there are additional inputs from the posterior ITC that carry largely contralateral positional information (Boussaoud et al., 1991). This may be what we observed in many CDt neurons.

Regardless of the underlying mechanism, the object- and position-selective information provides CDt neurons with a certain advantage in controlling motor behavior aiming at particular objects. However, its role is probably not exclusive. For example, the dorsal cortical visual pathway, which is considered to transmit mainly position-selective information, contains neurons that respond to visual objects selectively (Sereno and Maunsell, 1998; Murata et al., 2000; Janssen et al., 2008; Peng et al., 2008). The prefrontal cortex, which is thought to play an executive role in goal-directed motor behavior, contains neurons that encode both position and object information (Rao et al., 1997; Rainer et al., 1998). The position-object information in the parietal-frontal cortical areas may converge on to the frontal eye field (Peng et al., 2008) or area LIP (Sereno and Amador, 2006) from which saccadic commands may be issued. What then could distinguish between the basal ganglia mechanism and the parietal-frontal cortical mechanism? A difference may be found in the effect of familiarity. The object selectivity in the prefrontal cortical neurons increases with experience (Rainer and Miller, 2000). In con- 
trast, CDt neurons discriminate novel fractal objects equally as well as familiar fractal objects (Fig. $7 A, B$ ). Therefore, the CDt seems to have a built-in mechanism to precisely discriminate large sets of visual objects and their locations. However, the proposed difference between the cortical and basal ganglia mechanisms needs to be tested experimentally under the same behavioral context.

CDt neurons are also capable of inducing a motor behavior, specifically saccadic eye movements. This conclusion was obtained from two sets of data: (1) saccades were induced by electrical stimulation of the CDt, and (2) CDt neurons increase their activity before spontaneous saccades to objects. Each set of data provides deeper insights into the mechanisms and functions of the CDt.

The stimulation-induced saccades were likely due to activation of CDt neurons, because the threshold for evoking saccades was quite low, comparable with the threshold for the frontal eye field (FEF) (Bruce et al., 1985) or SC (Robinson, 1972), and was lower than the threshold for area LIP (Shibutani et al., 1984). More importantly, the sites effective for evoking saccades are largely confined in and adjacent to the CDt (Figs. 10, 11). Such scrutiny led to another intriguing finding: spatial organization within the CDt. By slightly changing the site of electrical stimulation, the vector (direction and amplitude) of the stimulationinduced saccade changed significantly (Fig. 11). Furthermore, the varying saccade vector roughly corresponded to the visual receptive fields of nearby $\mathrm{CDt}$ neurons. This suggests that the contralateral visual hemifield is represented in the CDt in a spatially heterogeneous manner and that the spatial organization may be useful for inducing saccades to specific locations.

The CDt stimulation induced saccades at variable timings (Fig. $10 B, C$ ), similarly to the stimulation of the caudate head in the cat (Kitama et al., 1991). This is in sharp contrast with the stimulation of the FEF (Robinson and Fuchs, 1969) or SC (Robinson, 1972), which evoked saccades with nearly constant latencies. It is as if the CDt stimulation opened a gate for saccades to be evoked based on signals from structures outside the CDt. In fact, the caudate nucleus, at least its anterior portion (head or body), is known to exert a facilitatory effect on the SC by removing the tonic inhibition from the SNr to the SC (Hikosaka et al., 2000). It is feasible that the CDt has the same mechanism. While the SC is disinhibited by the CDt stimulation, saccadic motor neurons in the SC would be activated if there are other excitatory inputs, say, from the FEF. Note that, if the CDt works as a gate for saccade initiation, the gating should be both position selective and object selective. In fact, the $\mathrm{SNr}-\mathrm{SC}$ connection carries positionselective signals: $\mathrm{SNr}$ neurons have restricted visual receptive fields (Hikosaka and Wurtz, 1983a) and project to the part of the SC with similar response fields (Hikosaka and Wurtz, 1983b). Furthermore, $\mathrm{SNr}$ neurons respond to fractals in an objectselective manner, although the selectivity is weaker than that of CDt neurons (our unpublished observation). Each CDt neuron, assuming that it has an inhibitory connection to the $\mathrm{SNr}$, would contribute to the opening of the gate, but only when the preferred object of the neuron is present (what) in the preferred position of the neuron (where). In this scenario, other excitatory inputs may not need to carry precise what-where information but should determine the precise timing of the saccade. Neurons in the FEF or LIP, which may provide such excitatory inputs, do carry what information (Sereno and Maunsell, 1998; Peng et al., 2008). However, according to a comparison using the same object selectivity index (see Materials and Methods), the object selectivity of CDt neurons (mean SI, 0.67 for familiar objects; 0.69 for novel objects) was considerably higher than the object selectivity of FEF neurons (mean SI, 0.25 ) or LIP neurons (mean SI, 0.35). We thus speculate that, for making saccades to visual objects, the CDtSNr-SC pathway may determine what, whereas the FEF/LIP-SC pathway may determine when. Where may be determined by both pathways.

Electrical stimulation of the CDt revealed that the CDt possesses a mechanism to guide saccade initiation. That the CDt actually uses the mechanism to guide saccades was suggested by our next observation that CDt neurons increased their activity before saccades to objects (Fig. 12). When there is only a single object, we may make a saccade to it regardless of what the object is. But when there are multiple objects, what information becomes critical because we need to choose one of them before making a saccade. Indeed, in such a multiple-object condition, CDt neurons often became active before saccades if they were directed to the preferred object of the neuron and if the object was present in the preferred location of the neuron (Fig. 12C). This finding suggests that individual CDt neurons control the initiation of saccades that are directed to particular objects.

The CDt is part of the basal ganglia, which are highly developed in the primate. There, we found highly object-selective and position-selective visual signals. Unexpected for such high visual sensitivity, the CDt has a remarkable motor function: its activation can induce saccadic eye movements. To our knowledge, this type of motor functionality has never been demonstrated in an area with sharp object selectivity. Therefore, the CDt may be uniquely situated to guide gaze accurately to selected visual objects in complex visual environments. When and how the CDt uses the mechanism to select visual objects will be addressed in future studies.

\section{References}

Beckstead RM, Edwards SB, Frankfurter A (1981) A comparison of the intranigral distribution of nigrotectal neurons labeled with horseradish peroxidase in the monkey, cat, and rat. J Neurosci 1:121-125.

Boussaoud D, Desimone R, Ungerleider LG (1991) Visual topography of area TEO in the macaque. J Comp Neurol 306:554-575.

Brown VJ, Desimone R, Mishkin M (1995) Responses of cells in the tail of the caudate nucleus during visual discrimination learning. J Neurophysiol 74:1083-1094.

Bruce CJ, Goldberg ME, Bushnell MC, Stanton GB (1985) Primate frontal eye fields. II. Physiological and anatomical correlates of electrically evoked eye movements. J Neurophysiol 54:714-734.

Caan W, Perrett DI, Rolls ET (1984) Responses of striatal neurons in the behaving monkey. 2. Visual processing in the caudal neostriatum. Brain Res 290:53-65.

DiCarlo JJ, Maunsell JH (2003) Anterior inferotemporal neurons of monkeys engaged in object recognition can be highly sensitive to object retinal position. J Neurophysiol 89:3264-3278.

Goodale MA, Milner AD (1992) Separate visual pathways for perception and action. Trends Neurosci 15:20-25.

Hays AV Jr, Richmond BJ, Optican LM (1982) Unix-based multipleprocess system, for real-time data acquisition and control. WESCON Conf Proc 1-10.

Hikosaka O, Wurtz RH (1983a) Visual and oculomotor functions of monkey substantia nigra pars reticulata. I. Relation of visual and auditory responses to saccades. J Neurophysiol 49:1230-1253.

Hikosaka O, Wurtz RH (1983b) Visual and oculomotor functions of monkey substantia nigra pars reticulata. IV. Relation of substantia nigra to superior colliculus. J Neurophysiol 49:1285-1301.

Hikosaka O, Sakamoto M, Usui S (1989) Functional properties of monkey caudate neurons. I. Activities related to saccadic eye movements. J Neurophysiol 61:780-798.

Hikosaka O, Takikawa Y, Kawagoe R (2000) Role of the basal ganglia in the control of purposive saccadic eye movements. Physiol Rev 80:953-978.

Janssen P, Srivastava S, Ombelet S, Orban GA (2008) Coding of shape and position in macaque lateral intraparietal area. J Neurosci 28:6679-6690. 
Karabelas AB, Moschovakis AK (1985) Nigral inhibitory termination on efferent neurons of the superior colliculus: an intracellular horseradish peroxidase study in the cat. J Comp Neurol 239:309-329.

Kitama T, Ohno T, Tanaka M, Tsubokawa H, Yoshida K (1991) Stimulation of the caudate nucleus induces contraversive saccadic eye movements as well as head turning in the cat. Neurosci Res 12:287-292.

Lehky SR, Peng X, McAdams CJ, Sereno AB (2008) Spatial modulation of primate inferotemporal responses by eye position. PLoS One 3:e3492.

Liu P, Basso MA (2008) Substantia nigra stimulation influences monkey superior colliculus neuronal activity bilaterally. J Neurophysiol 100: $1098-1112$.

Malpeli JG, Baker FH (1975) The representation of the visual field in the lateral geniculate nucleus of Macaca mulatta. J Comp Neurol 161:569594.

Middleton FA, Strick PL (1996) The temporal lobe is a target of output from the basal ganglia. Proc Natl Acad Sci U S A 93:8683-8687.

Mishkin M, Ungerleider LG, Macko KA (1983) Object vision and spatial vision: two cortical pathways. Trends Neurosci 6:414-417.

Miyashita Y, Higuchi S, Sakai K, Masui N (1991) Generation of fractal patterns for probing the visual memory. Neurosci Res 12:307-311.

Murata A, Gallese V, Luppino G, Kaseda M, Sakata H (2000) Selectivity for the shape, size, and orientation of objects for grasping in neurons of monkey parietal area AIP. J Neurophysiol 83:2580-2601.

Op De Beeck H, Vogels R (2000) Spatial sensitivity of macaque inferior temporal neurons. J Comp Neurol 426:505-518.

Parthasarathy HB, Schall JD, Graybiel AM (1992) Distributed but convergent ordering of corticostriatal projections: analysis of the frontal eye field and the supplementary eye field in the macaque monkey. J Neurosci 12:4468-4488.

Peng X, Sereno ME, Silva AK, Lehky SR, Sereno AB (2008) Shape selectivity in primate frontal eye field. J Neurophysiol 100:796-814.

Plenz D, Kitai ST (1998) Up and down states in striatal medium spiny neurons simultaneously recorded with spontaneous activity in fast-spiking interneurons studied in cortex-striatum-substantia nigra organotypic cultures. J Neurosci 18:266-283.

Rainer G, Miller EK (2000) Effects of visual experience on the representation of objects in the prefrontal cortex. Neuron 27:179-189.

Rainer G, Asaad WF, Miller EK (1998) Memory fields of neurons in the primate prefrontal cortex. Proc Natl Acad Sci U S A 95:15008-15013.

Rao SC, Rainer G, Miller EK (1997) Integration of what and where in the primate prefrontal cortex. Science 276:821-824.
Robinson DA (1972) Eye movements evoked by collicular stimulation in the alert monkey. Vision Res 12:1795-1808.

Robinson DA, Fuchs AF (1969) Eye movements evoked by stimulation of frontal eye fields. J Neurophysiol 32:637-648.

Saint-Cyr JA, Ungerleider LG, Desimone R (1990) Organization of visual cortical inputs to the striatum and subsequent outputs to the pallidonigral complex in the monkey. J Comp Neurol 298:129-156.

Saleem KS, Logothetis NK (2007) A combined MRI and histology atlas of the rhesus monkey brain. New York: Academic.

Selemon LD, Goldman-Rakic PS (1985) Longitudinal topography and interdigitation of corticostriatal projections in the rhesus monkey. J Neurosci 5:776-794.

Sereno AB, Amador SC (2006) Attention and memory-related responses of neurons in the lateral intraparietal area during spatial and shape-delayed match-to-sample tasks. J Neurophysiol 95:1078-1098.

Sereno AB, Lehky SR (2011) Population coding of visual space: comparison of spatial representations in dorsal and ventral pathways. Front Comput Neurosci 4:159.

Sereno AB, Maunsell JH (1998) Shape selectivity in primate lateral intraparietal cortex. Nature 395:500-503.

Shibutani H, Sakata H, Hyvärinen J (1984) Saccade and blinking evoked by microstimulation of the posterior parietal association cortex of the monkey. Exp Brain Res 55:1-8.

Shook BL, Schlag-Rey M, Schlag J (1991) Primate supplementary eye field. II. Comparative aspects of connections with the thalamus, corpus striatum, and related forebrain nuclei. J Comp Neurol 307:562-583.

Stanton GB, Goldberg ME, Bruce CJ (1988) Frontal eye field efferents in the macaque monkey: I. Subcortical pathways and topography of striatal and thalamic terminal fields. J Comp Neurol 271:473-492.

Tanaka K (1996) Inferotemporal cortex and object vision. Annu Rev Neurosci 19:109-139.

Van Hoesen GW, Yeterian EH, Lavizzo-Mourey R (1981) Widespread corticostriate projections from temporal cortex of the rhesus monkey. J Comp Neurol 199:205-219.

Webster MJ, Bachevalier J, Ungerleider LG (1995) Transient subcortical connections of inferior temporal areas TE and TEO in infant macaque monkeys. J Comp Neurol 352:213-226.

Yeterian EH, Van Hoesen GW (1978) Cortico-striate projections in the rhesus monkey: the organization of certain cortico-caudate connections. Brain Res 139:43-63. 